Draft Version OCtober 17, 2018

Preprint typeset using $\mathrm{IAT}_{\mathrm{E}} \mathrm{X}$ style emulateapj v. 5/2/11

\title{
MID-IR FORCAST/SOFIA OBSERVATIONS OF M82
}

\author{
T. Nikola ${ }^{1}$, T.L. Herter ${ }^{1}$, W.D. VaccA ${ }^{2}$, J.D. Adams ${ }^{1}$, J.M. De Buizer ${ }^{2}$, G.E. Gull ${ }^{1}$, C.P. Henderson ${ }^{1}$, L.D.

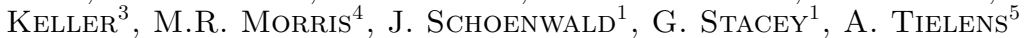 \\ Draft version October 17, 2018
}

\begin{abstract}
We present $75^{\prime \prime} \times 75^{\prime \prime}$ size maps of M82 at $6.4 \mu \mathrm{m}, 6.6 \mu \mathrm{m}, 7.7 \mu \mathrm{m}, 31.5 \mu \mathrm{m}$, and $37.1 \mu \mathrm{m}$ with a resolution of $\sim 4^{\prime \prime}$ that we have obtained with the mid-IR camera FORCAST on SOFIA. We find strong emission from the inner $60^{\prime \prime}(\sim 1 \mathrm{kpc})$ along the major axis, with the main peak $5^{\prime \prime}$ west-southwest of the nucleus and a secondary peak $4^{\prime \prime}$ east-northeast of the nucleus. The detailed morphology of the emission differs among the bands, which is likely due to different dust components dominating the continuum emission at short mid-IR wavelengths and long mid-IR wavelengths. We include SpitzerIRS and Herschel/PACS $70 \mu \mathrm{m}$ data to fit spectral energy distribution templates at both emission peaks. The best fitting templates have extinctions of $A_{V}=18$ and $A_{V}=9$ toward the main and secondary emission peak and we estimated a color temperature of $68 \mathrm{~K}$ at both peaks from the 31 $\mu \mathrm{m}$ and $37 \mu \mathrm{m}$ measurement. At the emission peaks the estimated dust masses are on the order of $10^{4} M_{\odot}$.

Subject headings: galaxies: ISM — galaxies: starburst — galaxies: individual (M82) — Infrared: ISM
\end{abstract}

\section{INTRODUCTION}

Over the last few $10^{8}$ yr a series of starbursts has been triggered in M82 de Grijs 2001; Mavva et al. 2006) due to its interaction with M81 and NGC 3077 (e.g. Appleton et al. 1981; Yun et al. 1994; Sun et al. 2005; de Mello et al. 2008). The most recent starburst $(\lesssim$ 50 Myr, e.g. Rieke et al. 1980; Satyapal et al. 1997) has created a stellar cluster at the center of M82. A ring of ionized gas that is enveloped by a molecular gas ring surrounds this cluster, and the starburst is likely fueled by gas that is funneled toward this region by a $\sim 1 \mathrm{kpc}\left(\sim 1^{\prime}\right)$ stellar bar (Telesco et al. 1991; Achtermann \& Lacy 1995; Wills et al. 2000; Greve et al. 2002). These gas rings, which appear between about $10^{\prime \prime}$ and $30^{\prime \prime}$ from the nucleus, could be gas swept up by a central expanding superbubble (Matsushita et al. 2000, 2005). High external gas pressure imposed on the molecular clouds probably drives the current starburst (Keto et al. 2005). This view is supported by the detection of warm molecular gas that is likely heated by dissipation of turbulence (Panuzzo et al. 2010).

The view to the center of M82 is obscured by patchy and high extinction (Satyapal et al. 1995; Förster Schreiber et al. 2001). The mid-infrared (midIR) wavelength regime, where the extinction is modest, is therefore used extensively to study the center of M82 (e.g. Rieke et al. 1980; Telesco et al. 1991; Förster Schreiber et al. 2001; Beirão et al. 2008). Individual star forming clouds that are located at the inner edge of the molecular gas ring or within the ionized gas ring have been observed in the mid-IR with large ground-

\footnotetext{
${ }^{1}$ Department of Astronomy, Cornell University, Ithaca, NY 14853, USA

${ }^{2}$ Universities Space Research Association, NASA Ames Research Center, MS 211-3, Moffett Field, CA 94035, USA

${ }^{3}$ Ithaca College, Ithaca, NY 14850, USA

${ }^{4}$ Department of Physics and Astronomy, University of California, Los Angeles, CA 90095-1547, USA

${ }^{5}$ Leiden Observatory, PO Box 9513, Leiden, 2300 RA, Netherlands
}

based observatories that provide (sub-) arcsecond resolution (Lipscy \& Plavchan 2004; Gandhi et al. 2011). Unfortunately, ground-based mid-IR observations are limited to a few telluric windows at $\lesssim 30 \mu \mathrm{m}$.

We have used the mid-IR camera FORCAST on SOFIA to study the central region of M82. FORCAST provides relatively high spatial resolution and also covers the entire 5-40 $\mu \mathrm{m}$ wavelength range. Emission from hot, small grains dominates the continuum at short midIR wavelengths while emission from warm, large grains dominates at long mid-IR wavelengths. A total wavelength coverage is therefore important to determine the detailed contribution of each dust component to the continuum emission and to estimate the heating of the dust.

We adopt a distance of $3.5 \mathrm{Mpc}$ for M82 (Dalcanton et al. 2009), so that $1^{\prime \prime}$ corresponds to $17.5 \mathrm{pc}$.

\section{OBSERVATION}

We observed M82 in the $6.35 \mu \mathrm{m}(\Delta \lambda=0.14 \mu \mathrm{m}), 6.61$ $\mu \mathrm{m}(\Delta \lambda=0.24 \mu \mathrm{m}), 7.71 \mu \mathrm{m}(\Delta \lambda=0.47 \mu \mathrm{m}), 31.5 \mu \mathrm{m}$ $(\Delta \lambda=5.7 \mu \mathrm{m})$, and $37.1 \mu \mathrm{m}(\Delta \lambda=3.3 \mu \mathrm{m})$ passband filters using FORCAST (Adams et al. 2010; Herter et al. 2012b) on SOFIA (Young et al. 2012).

FORCAST is a dual-channel camera with a shortwavelength channel $(5-25 \mu \mathrm{m})$ and a long-wavelength channel $(25-40 \mu \mathrm{m})$, allowing for simultaneous observations at two specific wavelengths by selecting bandpass filters in filter wheels. A dichroic beam-splitter directs the same field of view to both the short- and longwavelength cameras. To increase sensitivity, the beamsplitter can be either removed or replaced by a mirror during an observation. The effective field of view of the two 256 square arrays is $3.4^{\prime} \times 3.2^{\prime}$. After distortion correction, the pixel size is $0.768^{\prime \prime}$.

We observed M82 on 2010 December 1 in the two long wavelength bands without dichroic at an altitude of about 43000 feet. We used the on-chip chop-nod observing mode with a chopper throw and nod-distance of $120^{\prime \prime}$ and $90^{\prime \prime}$, respectively, and a chop frequency of $2 \mathrm{~Hz}$. 
In this mode the source is alway on the detector array. For the $37 \mu \mathrm{m}$ and $31 \mu \mathrm{m}$ observations we took seven and five integrations, respectively, with an on-source time of $60 \mathrm{~s}$ per integration. The observations in the $37 \mu \mathrm{m}$ band were taken at a zenith angle of about $58^{\circ}$.

On 2010 December 4, we obtained four integrations of M82 in the $6.6 \mu \mathrm{m}$ and $7.7 \mu \mathrm{m}$ bands and eight integrations in the $6.4 \mu \mathrm{m}$ band from an altitude of about 42000 feet. All bands were observed without beam-splitter, except for the $7.7 \mu \mathrm{m}$ band observations, which were observed simultaneously with the $31 \mu \mathrm{m}$ band. We observed again in the on-chip chop-nod mode, but with a chopper throw and nod-distance of $90^{\prime \prime}$ and a chop frequency of $5 \mathrm{~Hz}$. All bands were observed at a zenith angle of about $53^{\circ}$.

Since M82 is bright in all bands, we spatially registered the individual chop/nod integrations using a twodimensional Gaussian fit over the entire galaxy before co-adding the individual integrations. These pointingcorrections are about 3 pixels and improve the noise and the final maps. Due to the on-chip chop-nod observing mode the extent of the final images is about $75^{\prime \prime} \times 75^{\prime \prime}$. Since pointing accuracy and drifts were on the order of several arcseconds, we determined the absolute position of the M82 observation by comparing the $7.7 \mu \mathrm{m}$ FORCAST map with the $8 \mu \mathrm{m}$ Spitzer/IRAC map from the Spitzer archive.

The flux densities in all bands were color-corrected. The standard stars $\beta \mathrm{Gem}, \beta \mathrm{UMi}$, and $\mu \mathrm{UMa}$ were used for absolute flux density calibration and to estimate the beam size. Based on the uncertainty of flat fielding and the water vapor burden, as well as the variance of the flux density measurements, the uncertainty of the intrinsic flux density of the standard stars, and the fact that $\beta \mathrm{Gem}$ and $\beta \mathrm{UMi}$ are variable stars, we estimate a $3 \sigma$ uncertainty of $\sim 20 \%$ for the absolute calibration (Herter et al. 2012a, b) . Due to turbulent airflow across the telescope and pointing instabilities the observations are not diffraction limited. For the M82 observations, we estimate a beam size of about $4^{\prime \prime} \pm 0.5^{\prime \prime}$ for all the bands.

The original maps of the $6.4 \mu \mathrm{m}, 6.6 \mu \mathrm{m}$, and $7.7 \mu \mathrm{m}$ continuum had a lower signal-to-noise than the two longwavelength maps and we therefore smoothed them with a 2 pixel FWHM Gaussian kernel. The final flux densities are presented in Table 1

We include Herschel/PACS $70 \mu \mathrm{m}$ photometric data in our spectral energy distribution (SED) analysis below. Since processed and published PACS $70 \mu \mathrm{m}$ photometric data were unavailable, we used level 2.5 data product from the Herschel archive. The observations were carried out in scan-map mode at medium speed $\left(20^{\prime \prime} \mathrm{s}^{-1}\right)$ resulting in a smeared beam of about $5.46^{\prime \prime} \times 5.76^{\prime \prime}(\mathrm{PACS}$ Observer Manual). We extracted background-subtracted flux densities within a $6.8^{\prime \prime}$ aperture $(\sim 3.5$ PACS pixels) at three positions (see Table 1 for positions). The flux densities are $115 \pm 11 \mathrm{Jy}$ (main peak), $98 \pm 10 \mathrm{Jy}$ (secondary peak), and $116 \pm 11 \mathrm{Jy}$ (ridge). Within a radius of $35^{\prime \prime}$, similar to the entire region observed with FORCAST, the $70 \mu \mathrm{m}$ flux density is $1644 \pm 41 \mathrm{Jy}$.

\section{RESULTS}

3.1. Morphology
Figure 1 shows the distribution of the mid-IR emission in each of the observed bands. The $6.4 \mu \mathrm{m}, 6.6$ $\mu \mathrm{m}$, and $7.7 \mu \mathrm{m}$ emission is slightly more extended than the $31 \mu \mathrm{m}$ and $37 \mu \mathrm{m}$ emission. Also, the contrast in the morphology is higher in the $31 \mu \mathrm{m}$ and $37 \mu \mathrm{m}$ emission than in the short-wavelength bands, with the peaks being more pronounced. The main peak of all mid-IR distributions is $4.5^{\prime \prime}$ west-southwest from the dynamical center. Strong emission extends further to the west-southwest and "peaks" roughly $9^{\prime \prime}$ from the center ("western ridge"). A secondary peak is visible 4 " eastnortheast of the center (except at $6.6 \mu \mathrm{m}$ ). In the $7.7 \mu \mathrm{m}$ band, the emission at the secondary peak appears as two separate components. The positions of the main and secondary peaks coincide roughly with the position of the ionized ring or the inner edge of the molecular ring.

The $7.7 \mu \mathrm{m}$ FORCAST map is in excellent agreement with the Spitzer/IRAC $8 \mu \mathrm{m}$ map (Figure 1). Also, the FORCAST $31 \mu \mathrm{m}$ map agrees very well with the $30 \mu \mathrm{m}$ map obtained by Telesco et al. (1991) using the NASA Infrared Telescope Facility (IRTF), and the flux densities at the main and secondary peaks agree within the calibration uncertainty.

In Figure 2 we show a three-color image of M82, where we have combined the $6.6 \mu \mathrm{m}$ (blue), $31 \mu \mathrm{m}$ (green), and $37 \mu \mathrm{m}$ (red) bands. All colors are scaled linearly with flux density and start at the $3 \sigma$ level of the statistical background noise in each band. The image shows that the $6.6 \mu \mathrm{m}$ is more extended and that the secondary peak and western ridge are more pronounced in the two longwavelength bands.

Figure 3 shows the emission profiles along a $60^{\prime \prime}$ cut following the major axis of M82. The length and position of the cut is shown in Figure1. The profiles are in steps of 1 pixel and the flux densities are summed over $1 \times 5$ pixel strips perpendicular to the major axis and normalized to the emission peak. They emphasize that in the 31 $\mu \mathrm{m}$ and $37 \mu \mathrm{m}$ bands the ratio between the main and secondary peak is much stronger than in the three short mid-IR bands. In fact, the $6.6 \mu \mathrm{m}$ continuum only shows a gradient in the flux density from the main peak toward the position of the secondary peak. The emission from the "western ridge" is very pronounced in the $31 \mu \mathrm{m}$ and $37 \mu \mathrm{m}$ bands, less in the $7.7 \mu \mathrm{m}$ and $6.4 \mu \mathrm{m}$ maps, and barely noticeable in the $6.6 \mu \mathrm{m}$ map. The $31 \mu \mathrm{m}$ and 37 $\mu \mathrm{m}$ emission show a peak at this position that is stronger than the secondary peak.

Figure 3 also shows the flux density ratios along the major axis of M82. The profile of the $37 \mu \mathrm{m} / 6.6 \mu \mathrm{m}$ and $37 \mu \mathrm{m} / 7.7 \mu \mathrm{m}$ flux density ratios basically follows the profile of the $37 \mu \mathrm{m}$ flux density. In contrast, the flux density ratios of the $7.7 \mu \mathrm{m} / 6.6 \mu \mathrm{m}$ and $7.7 \mu \mathrm{m} / 6.4 \mu \mathrm{m}$ bands are fairly uniform along the major axis, with larger variations only at the edge of the profile, where the signal-to-noise becomes small. The profile of the $37 \mu \mathrm{m} / 31 \mu \mathrm{m}$ flux density ratio differs from all others. It is basically flat within $\pm 10^{\prime \prime}$ of the center of M82 and then increases on either side, while the flux density ratios of all other bands either decrease or stay constant with distance from the center. This indicates a higher color temperature of the large grains in the inner region than in the outer region. Also, the difference of the profiles between the three short midIR bands and two long mid-IR bands indicate that they trace different dust components with spatially distinct 
excitation conditions.

\subsection{Mid-IR Spectral Energy Distribution}

Siebenmorgen \& Krügel (2007) $)^{6}$ have created template SEDs for starbursts as a function of the total luminosity $(L($ tot $))$, optical extinction, contribution to the total luminosity by OB stars, and radius of the nuclear starburst, covering the wavelength range $0.03-2000 \mu \mathrm{m}$. We use the FORCAST and PACS $70 \mu \mathrm{m}$ data points as well as low-resolution Spitzer-IRS spectra between $5.3 \mu \mathrm{m}$ to $12.8 \mu \mathrm{m}$ from region 2 and 3 of Beirão et al. (2008) to find the SED template that fits the data in the two midIR emission peaks within a $6.8^{\prime \prime} \times 6.8^{\prime \prime}(\sim 115 \times 115 \mathrm{pc})$ area, corresponding to $\sim 9 \times 9$ FORCAST pixels. The lower size limit of the area is given by the Spitzer observation. Since this area is much smaller than the smallest nuclear radius in the SED template grid (350 pc), we constrained our search algorithm to that template series and allow it to fit an additional scaling factor. Figure 4 shows the data points and the best-fitting SED template at the main and secondary peaks. It includes three data points from Telesco et al. (1991), multiplied by a factor of two, which corresponds to the flux density ratio within a $6.8^{\prime \prime}$ and $4.5^{\prime \prime}$ aperture in a FORCAST band at the peaks. The flux densities at $70 \mu \mathrm{m}$ and $37 \mu \mathrm{m}$ at the main and secondary peaks are very similar and the 70 $\mu \mathrm{m}$ flux density is thus crucial to constrain the SED fit.

For the main mid-IR emission peak, the best-fitting SED template has a total luminosity of $L_{\mathrm{MP}}($ tot $)=$ $10^{11.2} L_{\odot}$, an OB luminosity fraction of $40 \%$, an extinction of $A_{V}=18$, and a hydrogen density of $n=5 \times 10^{3}$ $\mathrm{cm}^{-3}$ in the hot spots. The template requires an additional scaling factor of 0.041 , which is of the order of the ratio of observed to the template area, and lowers the total luminosity to $L_{\mathrm{MP}}($ tot $)=6.7 \times 10^{9} L_{\odot}$. The best fitting model for the secondary emission peak has a total luminosity of $L_{\mathrm{SP}}($ tot $)=10^{10.7} L_{\odot}$, an $\mathrm{OB}$ luminosity fraction of $40 \%$, an extinction of $A_{V}=9$, a hydrogen density of $n=5 \times 10^{3} \mathrm{~cm}^{-3}$, and requires an additional scaling factor of 0.11 , which reduces the total luminosity to $L_{\mathrm{SP}}($ tot $)=5.7 \times 10^{9} L_{\odot}$.

Due to limited resolution of the SED template parameters we estimate the derived extinction to be within a factor of two. Both values are lower than the extinction reported by Förster Schreiber et al. (2001) for the entire central region $\left(A_{\mathrm{V}}=52 \pm 17\right)$. This discrepancy could be due to the patchy extinction in the central region (Satvapal et al. 1995; Lipscy \& Plavchan 2004; Gandhi et al. 2011).

\subsubsection{Extinction}

The extinction laws between 3 and $10 \mu \mathrm{m}$ toward the center of M82 and the Galactic center (GC) are similar (Förster Schreiber et al. 2001), and for the GC it is best modeled with $R_{V}=5.5$ (Draine 2003). To estimate the extinction and emissivity at the FORCAST bands we therefore use the GC extinction law as modeled by Li \& Draine (2001) and Weingartner \& Draine (2001) 7 with $R_{V}=5.5$, a gas-to-dust mass ratio of 105 , a dust grain mass per hydrogen atom of $m_{d}=2.2 \times 10^{-26} \mathrm{~g} / \mathrm{H}$,

6 www.eso.org/ rsiebenm/sb_models/

7 www.astro.princeton.edu/ ${ }^{\sim}$ draine/dust/dustmix.html
$N(\mathrm{H}) / A_{V}=1.37 \times 10^{21} \mathrm{~cm}^{-2} \operatorname{mag}^{-1}($ with $N(\mathrm{H})=$ $N(\mathrm{HI})+N\left(\mathrm{H}_{2}\right)$ ), and an emissivity index of $\beta=1.79$ for the $31 \mu \mathrm{m}$ and $37 \mu \mathrm{m}$ bands. The mass absorption coefficient and albedo at 31.62 and $37.15 \mu \mathrm{m}$ are $\kappa=385.2$ $\mathrm{cm}^{2} \mathrm{~g}^{-1}$ and 0.0007 , and $\kappa=288.8 \mathrm{~cm}^{2} \mathrm{~g}^{-1}$ and 0.0006 , respectively. Using the relation $\kappa_{\text {abs }}(\lambda)=0.4 \ln 10(1-$ albedo $) \times\left[A(\lambda) / N_{\mathrm{H}}\right] / m_{\text {dust }} \mathrm{cm}^{2} \mathrm{~g}^{-1}(\mathrm{Li} \&$ Draine 2001) leads to $A(37) / A_{V}=9.46 \times 10^{-3}$ and $A(31) / A_{V}=1.26 \times$ $10^{-2}$, resulting in $\tau(37 \mu \mathrm{m})=0.16$ and $\tau(31 \mu \mathrm{m})=0.21$ at the main emission peak, respectively. At the secondary emission peak the opacities are $\tau(37 \mu \mathrm{m})=0.08$ and $\tau(31 \mu \mathrm{m})=0.11$

\subsubsection{Dust Mass}

We use three different methods to estimate the dust masses at the main and secondary emission peaks within $6.8^{\prime \prime} \times 6.8^{\prime \prime}$.

Applying the extinction laws from the previous section we estimate the gas mass using $M_{\mathrm{g}}=\mu \times m_{\mathrm{H}} \times N(\mathrm{H}) \times$ Area, where $\mu=1.4$ is the mean atomic mass per hydrogen, $m_{\mathrm{H}}$ is the mass of atomic hydrogen, and $N(\mathrm{H})$ is the total hydrogen column density. We obtain a total gas mass of $M_{\mathrm{g}}(\mathrm{MP})=3.8 \times 10^{6} M_{\odot}$ at the main peak and $M_{\mathrm{g}}(\mathrm{SP})=1.9 \times 10^{6} M_{\odot}$ at the secondary peak. Using a gas-to-dust ratio of 105 this gives dust masses of $M_{\mathrm{d}}(\mathrm{MP})=3.6 \times 10^{4} M_{\odot}$ and $M_{\mathrm{d}}(\mathrm{SP})=1.8 \times 10^{4} M_{\odot}$ at the main and secondary peak, respectively. Due to the uncertainty of the extinction, the dust masses can vary by a factor of two.

The dust mass can also be estimated by $M_{\mathrm{d}}=1 / \kappa_{\text {abs }} \times$ $F_{\nu}(\lambda) \times D^{2} / B(\lambda, T)$, where $\kappa_{\text {abs }}$ is the mass absorption coefficient, $F_{\nu}$ is the flux density, $D$ is the distance to M82, and $B(\lambda, T)$ is the Planck function at wavelength $\lambda$ and temperature $T$. Using the previously estimated opacities for $31 \mu \mathrm{m}$ and $37 \mu \mathrm{m}$ and a modified blackbody function we estimate a color temperature of $68 \pm 10 \mathrm{~K}$ at both emission peaks. The resulting dust masses are $1.15 \times 10^{4} M_{\odot}$ and $7.9 \times 10^{3} M_{\odot}$ at the main and secondary peaks, respectively. The uncertainty of the masses is within a factor of four.

Sanders et al. (1991) estimate the dust mass using the relation $M_{\mathrm{d}}=\left(L(\mathrm{FIR}) / 10^{8} L_{\odot}\right) \times\left(40 \mathrm{~K} / T_{\mathrm{d}}\right)^{5} 10^{4} M_{\odot}$, where $T_{\mathrm{d}}$ is the dust temperature and $L(\mathrm{FIR})$ is the far-IR luminosity in the range between 40 and 500 $\mu \mathrm{m}$. From the best fitting model SED we determine a $L_{\mathrm{MP}}(\mathrm{FIR})=3.08 \times 10^{9} L_{\odot}$ at the main peak and $L_{\mathrm{SP}}(\mathrm{FIR})=2.52 \times 10^{9} L_{\odot}$ at the secondary peak. Using the color temperatures derived above we obtain dust masses of $M_{\mathrm{d}}(\mathrm{MP})=2.2 \times 10^{4} M_{\odot}$ and $M_{\mathrm{d}}(\mathrm{SP})=$ $1.8 \times 10^{4} M_{\odot}$ at the main and secondary peak. These dust masses are good to a factor of three.

High resolution $\mathrm{CO}$ measurements with a $4.2^{\prime \prime}$ aperture suggest molecular hydrogen column densities of $N\left(\mathrm{H}_{2}\right) \approx$ $(4--10) \times 10^{22} \mathrm{~cm}^{-2}$ at our main peak and $N\left(\mathrm{H}_{2}\right) \approx 4 \times$ $10^{22} \mathrm{~cm}^{-2}$ at our secondary peak (Weiß et al. 2001). So, the enclosed gas masses, including He and other heavy elements, are $(1.7--4.2) \times 10^{6} M_{\odot}$. Assuming a gas-todust ratio of 105 this yields dust masses between (1.6$-4) \times 10^{4} M_{\odot}$, in agreement with our calculations above.

\section{SUMMARY}

We have presented the first results of mid-IR observations of M82 obtained with FORCAST on SOFIA. M82 
was observed in the $6.4 \mu \mathrm{m}, 6.6 \mu \mathrm{m}, 7.7 \mu \mathrm{m}, 31.5 \mu \mathrm{m}$, and $37.1 \mu \mathrm{m}$ bands. The observations cover a $75^{\prime \prime} \times 75^{\prime \prime}$ region. All bands show a strong peak located $4.5^{\prime \prime}$ westsouthwest of the kinematic center of M82. A secondary peak $4^{\prime \prime}$ east-northeast of the nucleus is seen in the 6.4 $\mu \mathrm{m}, 7.7 \mu \mathrm{m}, 31.5 \mu \mathrm{m}$, and $37.1 \mu \mathrm{m}$ bands, but not in the $6.6 \mu \mathrm{m}$ continuum. The profiles of the flux density ratios over $60^{\prime \prime}$ along the major axis indicates that the emission at the three short mid-IR bands is dominated by a different dust component than the emission at the two long mid-IR bands. We fitted SED templates to the FORCAST data combined with PACS $70 \mu \mathrm{m}$ and low-resolution Spitzer-IRS spectra and estimated extinctions of $A_{V}=18$ and $A_{V}=9$ toward the main and secondary peaks and a dust color temperature of $68 \mathrm{~K}$ in both peaks. The dust masses at the locations of the emission peaks are on the order $10^{4} M_{\odot}$.

We thank SOFIA telescope engineering and operations team, as well as the SOFIA flight crews and mission operations team for their support. This work is based on observations made with FORCAST on the NASA/DLR Stratospheric Observatory for Infrared Astronomy (SOFIA). SOFIA science mission operations are conducted jointly by the Universities Space Research Association, Inc. (USRA), under NASA contract NAS297001, and the Deutsches SOFIA Institut (DSI) under DLR contract 50 OK 0901. Financial support for FORCAST was provided to Cornell by NASA through award 8500-98-014 issued by USRA. This work is based in part on observations made with the Spitzer Space Telescope, obtained from the NASA/ IPAC Infrared Science Archive, both of which are operated by the Jet Propulsion Laboratory, California Institute of Technology under a contract with the National Aeronautics and Space Administration. This research has made use of NASAs Astrophysics Data System Abstract Service.

\section{REFERENCES}

Achtermann, J. M., \& Lacy, J. H. 1995, ApJ, 439, 163

Adams, J. D., et al. 2010, in Society of Photo-Optical Instrumentation Engineers (SPIE) Conference Series, Vol. 7735, Society of Photo-Optical Instrumentation Engineers (SPIE)

Conference Series
Appleton, P. N., Davies, R. D., \& Stephenson, R. J. 1981 MNRAS, 195, 327

Beirão, P., et al. 2008, ApJ, 676, 304

Dalcanton, J. J., et al. 2009, ApJS, 183, 67

de Grijs, R. 2001, Astronomy and Geophysics, 42, 040000 de Mello, D. F., Smith, L. J., Sabbi, E., Gallagher, J. S.,

Mountain, M., \& Harbeck, D. R. 2008, AJ, 135, 548

Draine, B. T. 2003, ARA\&A, 41, 241

Förster Schreiber, N. M., Genzel, R., Lutz, D., Kunze, D., \& Sternberg, A. 2001, ApJ, 552, 544

Gandhi, P., Isobe, N., Birkinshaw, M., Worrall, D. M., Sakon, I. Iwasawa, K., \& Bamba, A. 2011, PASJ, 63, 505

Greve, A., Wills, K. A., Neininger, N., \& Pedlar, A. 2002, A\&A, 383,56

Herter, T. L., Vacca, W. D., \& D., A. J. 2012a, in prep.

Herter, T. L., et al. 2012b, ApJ, this volume

Keto, E., Ho, L. C., \& Lo, K.-Y. 2005, ApJ, 635, 1062

Li, A., \& Draine, B. T. 2001, ApJ, 554, 778

Lipscy, S. J., \& Plavchan, P. 2004, ApJ, 603, 82

Matsushita, S., Kawabe, R., Kohno, K., Matsumoto, H., Tsuru, T. G., \& Vila-Vilaró, B. 2005, ApJ, 618, 712

Matsushita, S., Kawabe, R., Matsumoto, H., Tsuru, T. G., Kohno, K., Morita, K.-I., Okumura, S. K., \& Vila-Vilaró, B. 2000, ApJ, 545, L107

Mayya, Y. D., Bressan, A., Carrasco, L., \& Hernandez-Martinez, L. 2006, ApJ, 649, 172

Panuzzo, P., et al. 2010, A\&A, 518, L37+

Rieke, G. H., Lebofsky, M. J., Thompson, R. I., Low, F. J., \& Tokunaga, A. T. 1980, ApJ, 238, 24

Sanders, D. B., Scoville, N. Z., \& Soifer, B. T. 1991, ApJ, 370, 158

Satyapal, S., Watson, D. M., Pipher, J. L., Forrest, W. J.,

Greenhouse, M. A., Smith, H. A., Fischer, J., \& Woodward, C. E. 1997, ApJ, 483, 148

Satyapal, S., et al. 1995, ApJ, 448, 611

Siebenmorgen, R., \& Krügel, E. 2007, A\&A, 461, 445

Sun, W.-H., et al. 2005, ApJ, 630, L133

Telesco, C. M., Joy, M., Dietz, K., Decher, R., \& Campins, H. 1991, ApJ, 369, 135

Weingartner, J. C., \& Draine, B. T. 2001, ApJ, 548, 296

Weiß, A., Neininger, N., Hüttemeister, S., \& Klein, U. 2001, A\&A, 365, 571

Wills, K. A., Das, M., Pedlar, A., Muxlow, T. W. B., \& Robinson, T. G. 2000, MNRAS, 316, 33

Young, E. T., et al. 2012, ApJ, this volume

Yun, M. S., Ho, P. T. P., \& Lo, K. Y. 1994, Nature, 372, 530 
Table 1

M82 FORCAST Flux Densities

\begin{tabular}{|c|c|c|c|c|c|c|c|}
\hline $\begin{array}{l}\text { Band } \\
(\mu \mathrm{m})\end{array}$ & $\begin{array}{c}S_{\text {peak }}^{\mathrm{a}} \\
\left(\text { Jy pixel }^{-1}\right)\end{array}$ & $\begin{array}{c}S_{\mathrm{mp}}^{\mathrm{b}, \mathrm{c}} \\
(\mathrm{Jy})\end{array}$ & $\begin{array}{l}S_{\mathrm{sp}}^{\mathrm{b}, \mathrm{d}} \\
(\mathrm{Jy})\end{array}$ & $\begin{array}{c}S_{\mathrm{wr}}^{\mathrm{b}, \mathrm{e}} \\
(\mathrm{Jy})\end{array}$ & $\begin{array}{l}S_{\mathrm{mp}}^{\mathrm{f}, \mathrm{c}} \\
(\mathrm{Jy})\end{array}$ & $\begin{array}{l}S_{\mathrm{sp}}^{\mathrm{f}, \mathrm{d}} \\
(\mathrm{Jy})\end{array}$ & $\begin{array}{c}S(\text { Total Map })^{\mathrm{g}} \\
(\mathrm{Jy})\end{array}$ \\
\hline 6.4 & $0.112 \pm 0.007$ & $3.42 \pm 0.23$ & $2.96 \pm 0.20$ & $2.75 \pm 0.18$ & $6.93 \pm 0.46$ & $6.02 \pm 0.40$ & $68 \pm 5$ \\
\hline 6.6 & $0.047 \pm 0.003$ & $1.40 \pm 0.09$ & $1.21 \pm 0.08$ & $1.17 \pm 0.08$ & $2.90 \pm 0.19$ & $2.52 \pm 0.17$ & $32 \pm 2$ \\
\hline 7.7 & $0.141 \pm 0.009$ & $4.12 \pm 0.28$ & $3.41 \pm 0.23$ & $3.20 \pm 0.21$ & $8.16 \pm 0.54$ & $6.94 \pm 0.46$ & $75 \pm 5$ \\
\hline 31.5 & $1.86 \pm 0.12$ & $57.1 \pm 3.8$ & $40.6 \pm 2.7$ & $46.5 \pm 3.1$ & $110.0 \pm 7.3$ & $79.7 \pm 5.3$ & $676 \pm 45$ \\
\hline 37.1 & $2.42 \pm 0.16$ & $74.5 \pm 5.0$ & $51.5 \pm 3.4$ & $61.4 \pm 4.1$ & $143.9 \pm 9.6$ & $102.2 \pm 6.8$ & $891 \pm 59$ \\
\hline
\end{tabular}

a Pixel size: $0.768^{\prime \prime}$.

b Within $6 \times 6$ pixels, corresponding to a $4.6^{\prime \prime} \times 4.6^{\prime \prime}$ region.

c Main peak: $09^{h} 55^{m} 51.28^{s},+69^{\circ} 40^{\prime} 45.5^{\prime \prime}$

d Secondary peak: $09^{h} 55^{m} 52.68^{s},+69^{\circ} 40^{\prime} 48.5^{\prime \prime}$

e Western Ridge: $09^{h} 55^{m} 50.47^{s},+69^{\circ} 40^{\prime} 43.9^{\prime \prime}$

$\mathrm{f}$ Within $9 \times 9$ pixels, corresponding to a $6.8^{\prime \prime} \times 6.8^{\prime \prime}$ region.

g Within $50^{\prime \prime} \times 75^{\prime \prime}(65 \times 98$ pixel $)$ region around center of M82 

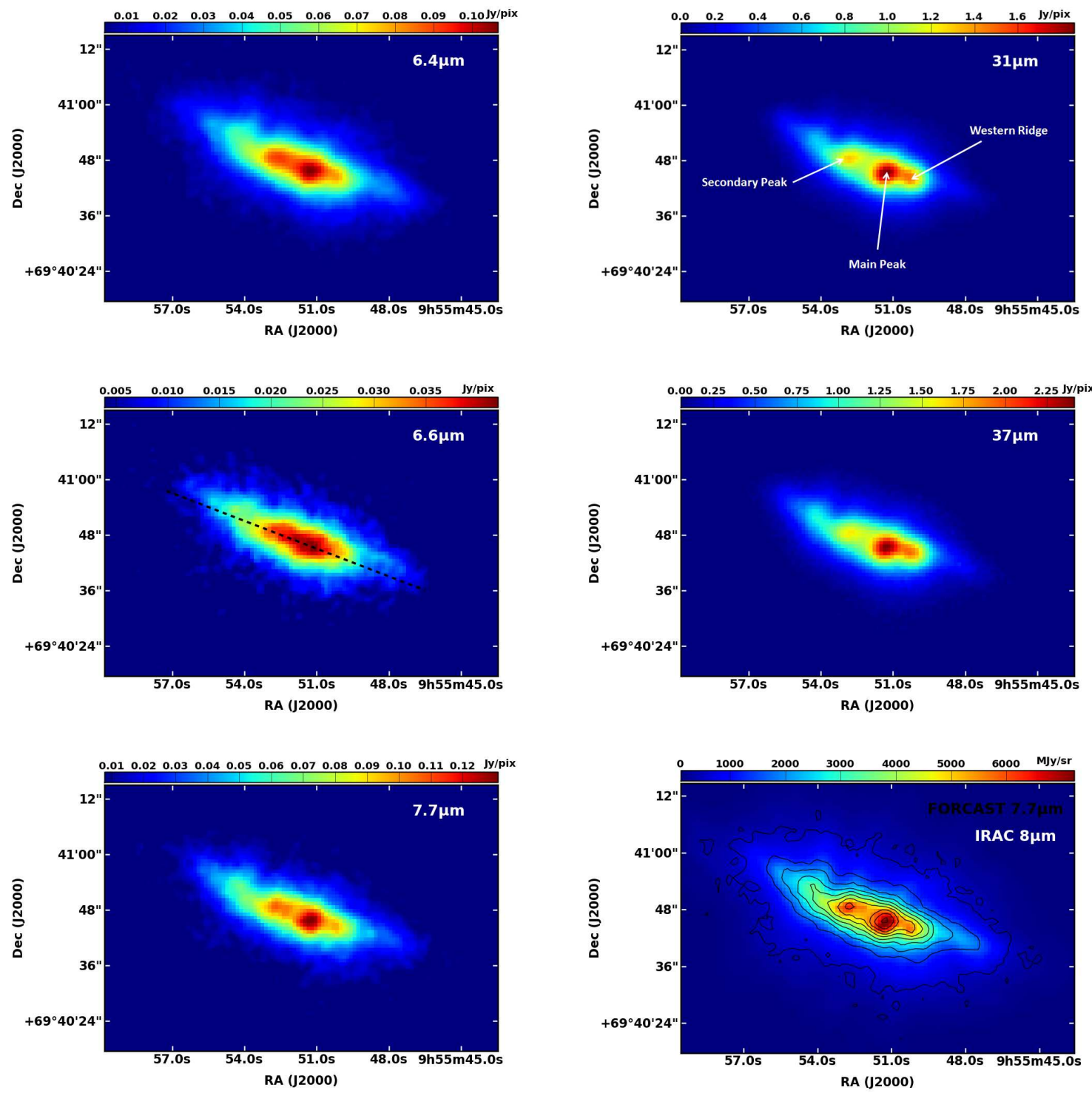

Figure 1. Maps of M82 in the FORCAST bands in units of Jy pixel ${ }^{-1}$ at $6.4 \mu \mathrm{m}$ (top left), $6.6 \mu \mathrm{m}$ (mid left), $7.7 \mu \mathrm{m}$ (bottom left), 31.5 $\mu \mathrm{m}$ (top right), and $37.1 \mu \mathrm{m}$ (mid right). Bottom right: IRAC band $4(8 \mu \mathrm{m})$ map (in MJy sr ${ }^{-1}$ ) overplotted with FORCAST $7.7 \mu \mathrm{m}$ contours . The color scale is linear and starts at the $3 \sigma$ level of the statistical background noise $(0.009 \mathrm{Jy}$ at 6.4 and $6.6 \mu \mathrm{m}, 0.018 \mathrm{Jy}$ at $7.7 \mu \mathrm{m}, 0.042 \mathrm{Jy}$ at $31.5 \mu \mathrm{m}$, and $0.051 \mathrm{Jy}$ at $37.1 \mu \mathrm{m}$ ). The dashed line in the $6.6 \mu \mathrm{m}$ map (mid left) indicates the position of the profiles shown in Figure 3 


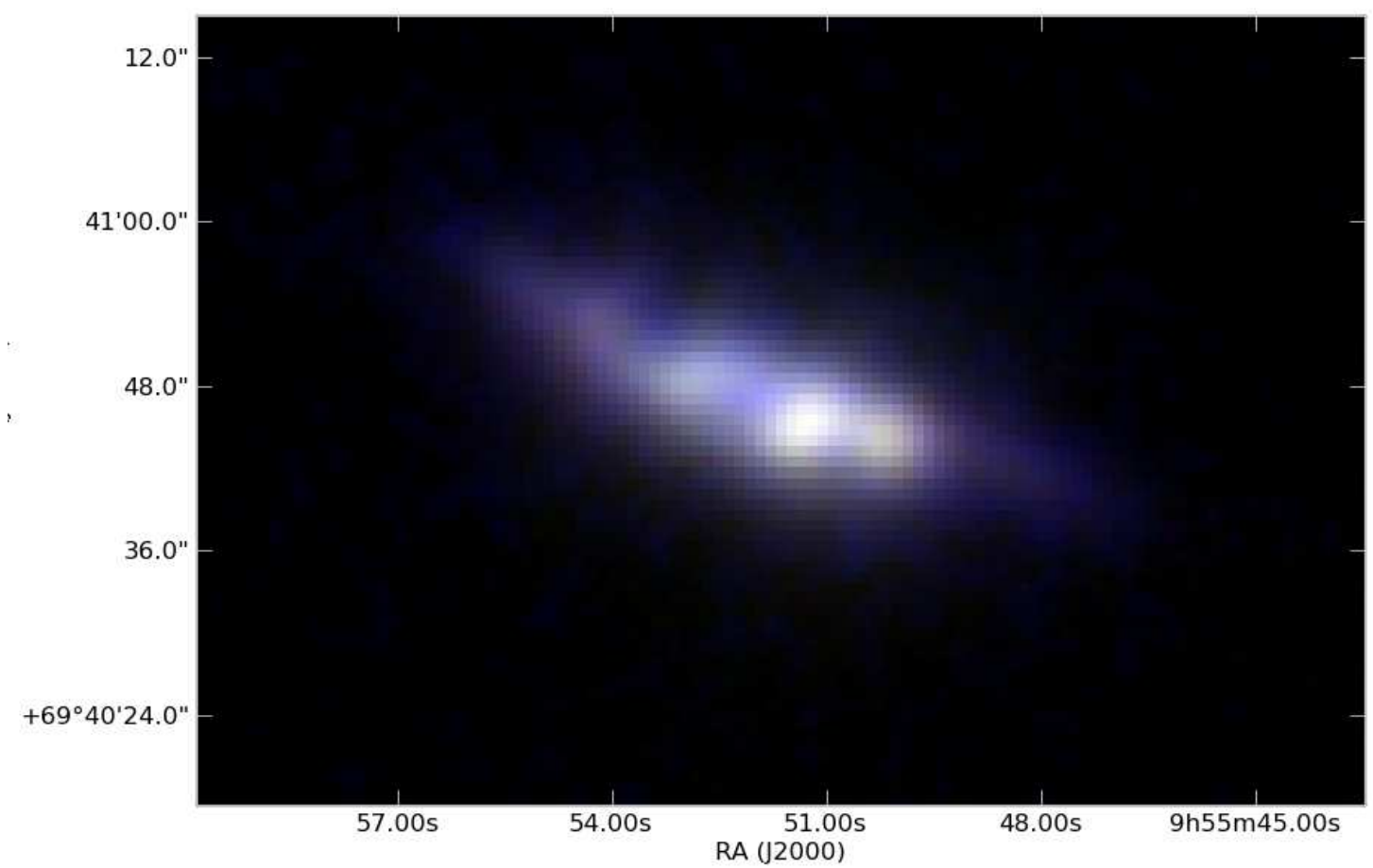

Figure 2. Three color image of M82, with the FORCAST $6.6 \mu \mathrm{m}$ as red, $31.5 \mu \mathrm{m}$ as green, and $37.1 \mu \mathrm{m}$ as blue. All bands are linearly scaled, starting from $3 \sigma$ of the statistical background noise, before combined. 

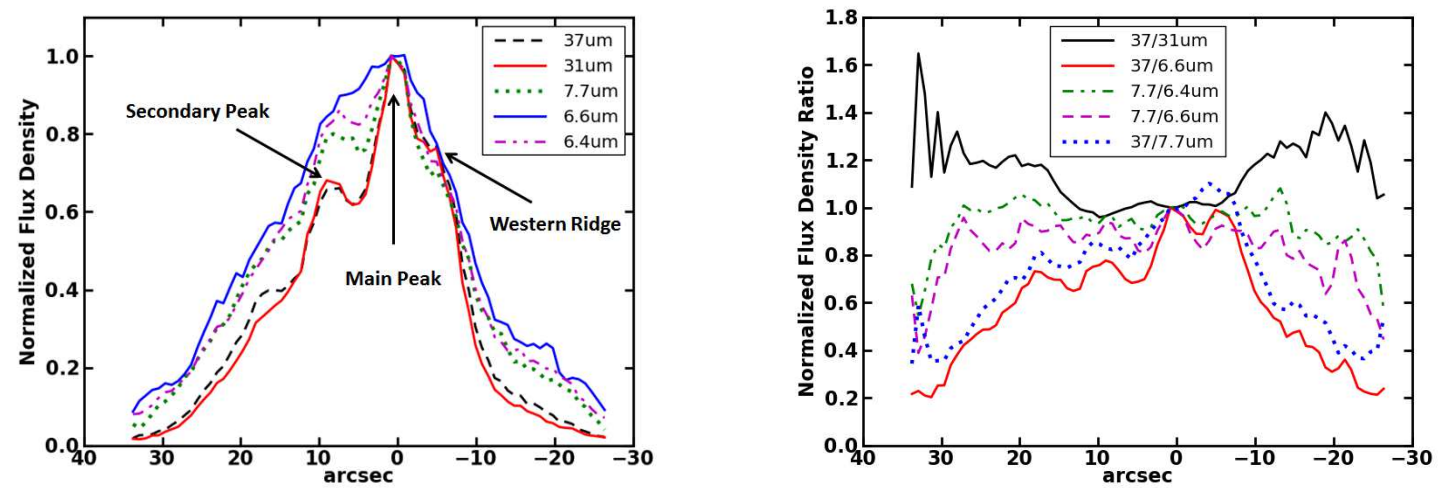

Figure 3. Flux densities (left) and flux density ratios (right), normalized to the value at the main peak position, along the major axis of M82. The reference position is the main peak, distances are in arcsec, and positive distance is toward the northeast. Flux densities are summed over $1 \times 5$ pixels perpendicular to the major axis. 
Mid-IR FORCAST/SOFIA Observations of M82
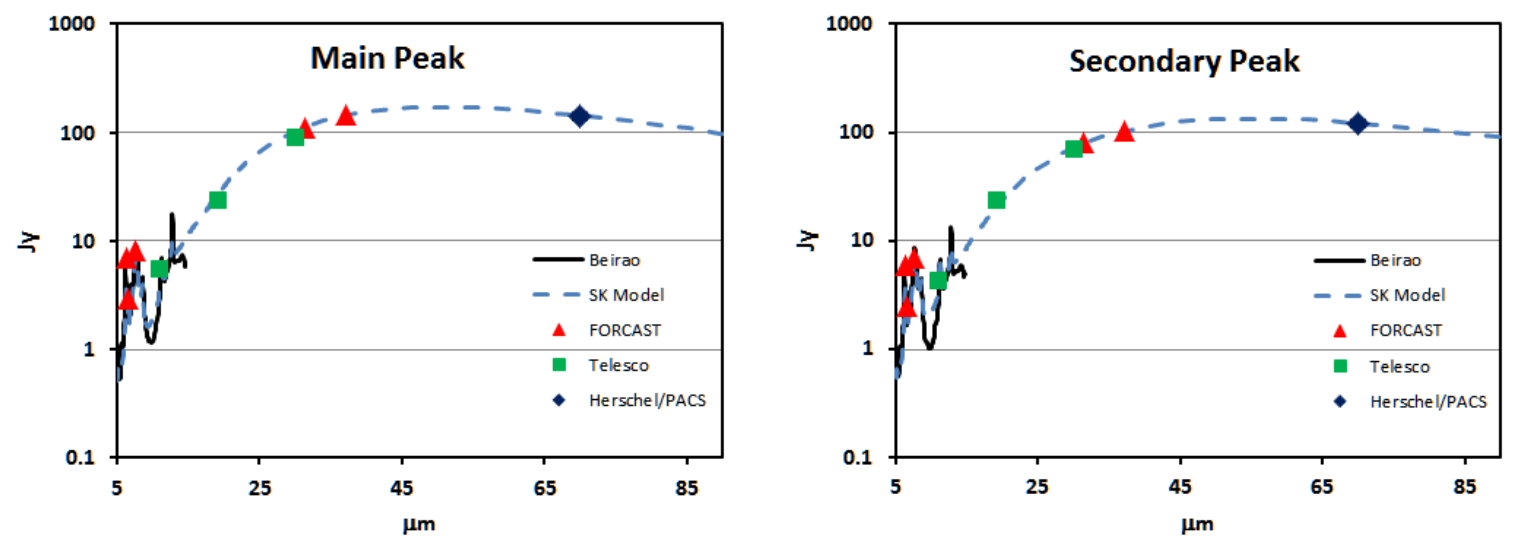

Figure 4. Mid-IR SED of the main peak (left) and secondary peak (right). The solid (black) line is the low-resolution Spitzer/IRS spectrum (Beirão et al. 2008), filled triangles (red) are the FORCAST observations, filled diamond (blue) is the Herschel/PACS $70 \mu \mathrm{m}$ observation, filled squares (green) are IRTF observations (Telesco et al.|[1991), multiplied by a factor of two (see the text), dashed line (blue) is the Siebenmorgen \& Kruegel SED model|Siebenmorgen \& Krügel (2007). The error bars are smaller than the symbols. 